\title{
Emission Intensity in the Hydrogen Atom Calculated from a Non-Probabilistic Approach to the Electron Transitions
}

\author{
Stanisław Olszewski \\ Institute of Physical Chemistry, Polish Academy of Sciences, Warsaw, Poland \\ Email: olsz@ichf.edu.pl
}

Received 10 March 2016; accepted 26 April 2016; published 29 April 2016

Copyright (C) 2016 by author and Scientific Research Publishing Inc.

This work is licensed under the Creative Commons Attribution International License (CC BY).

http://creativecommons.org/licenses/by/4.0/

c) (i) Open Access

\begin{abstract}
Quantum aspects of the Joule-Lenz law for the transmission of energy allowed us to calculate the time rate of energy transitions between the quantum states of the hydrogen atom in a fully nonprobabilistic way. The calculation has been extended to all transitions between $p$ and $s$ states having main quantum numbers not exceeding 6 . An evident similarity between the intensity pattern obtained from the Joule-Lenz law and the corresponding quantum-mechanical transition probabilities has been shown.
\end{abstract}

\section{Keywords}

Time Intervals for the Electron Transitions in the Hydrogen Atom, Non-Probabilistic Theory of Energy Emission in the Atom, Comparison of the Emission Intensities with the Quantum-Mechanical Transition Probabilities

\section{Introduction}

Since the very beginning of quantum theory the transition rate of energy connected with the occupation change of quantum states has been considered on a combined probabilistic-and-statistical footing [1]-[3]. Another formal probabilistic calculations of the rate of energy emitted in course of the electron transitions could be done on the basis of quantum mechanics; see e.g. [4] [5]. Experimentally a roughly precise measurement of the time of a single transition between two quantum levels seems to be hardly possible because of an extremely short interval of time expected to be associated with the transition phenomenon. In reality any experiment has its finite duration, so beyond of a short time of transition a whole electron population of transitions should be considered before the time of a single transition can be derived and estimated. 
However, the problem could obtain a new approach if the quantum background coupled with the Joule--Lenz law of the energy emission is taken into account. In this case the quantum aspects of that law discovered recently [6] [7] allowed us to make a step towards an understanding of the radiation theory which is much different in its character than a search for transition probabilities between the quantum states.

In effect we can calculate the transition time of a single electron particle between two quantum states. Further connection of such time with the energy rate of radiation becomes a simple task. An auxiliary component of this theory is the fact that it can be compared with the quantum-mechanical calculations giving a rather satisfactory assessment for the new, i.e. non-probabilistic, results.

This is so because the ratio of the intensities of two spectroscopic lines gives in fact the ratio of transition probabilities [8]. In emission spectra, the simplest conditions of excitation are those in which the excited states of the atoms are approximately in thermal equilibrium and the number of atoms in any given state is proportional to the Boltzmann factor. Usually in considering the ratio of intensities of two lines without specifying conditions, one practically assumes the temperature equilibrium at infinite temperature so that the Boltzmann factor is equal to unity. This assumption is realized especially well when the transitions originate from levels whose energies differ little from one another. Experimentally in flames and in certain parts of electric arcs the excitation corresponds approximately to thermal equilibrium, on the other hand in glow discharges the conditions of excitation are more complicated and it is not always possible to connect observed intensities with transition probabilities. However, if two lines have a common upper level their intensities will always be in the ratio of their transition probabilities [8].

In the present calculations of the changes of quantum states only the energy and time are involved. Therefore there is no reference to the selection rules of transitions given by such parameters like, for example, the orbital angular momentum.

\section{Time Intervals Considered instead of Transition Probabilities}

A characteristic step of a later Bohr's approach to the atomic spectra was a poposal of the Fourier analysis of the displacement vector associated with the position change of the particle submitted to transition [9]. This analysis was expected to give the probabilities of transitions between quantum states. But in our opinion a much more practical step than the displacement analysis is to examine the balance of time necessary to perform a transition. In fact this balance can be represented with the aid of the transition energy, too, because the components entering the balance of time can be defined with aid of the components of the transition energy. A complementary relation between energy and time becomes here especially of use if we note that the energy intervals are much more easy to calculate than the intervals of time.

In effect, because any elementary interval of energy has its corresponding interval of time, these elementary time intervals can be added together into full intervals necessary to be considered in description of a given quantum process. In result the rate of the electron transitions between rather distant quantum levels could be calculated as a function of the elementary intervals of energy. In the present paper we do such time analysis and apply it in calculating the rate of electron transitions in the hydrogen atom. Before we do that, the elementary properties of both energy and time entering the transitions will be represented.

\section{Elementary and Combined Transitions and Their Properties}

Elementary transition is that between two neighbouring quantum levels, say $n+1$ and $n$. Beginning with $n=1$ we obtain

$$
\begin{aligned}
& E_{2}-E_{1}=\Delta E_{1}, \\
& E_{3}-E_{2}=\Delta E_{2}, \\
& E_{4}-E_{3}=\Delta E_{3}, \cdots,
\end{aligned}
$$

in general

$$
E_{n+1}-E_{n}=\Delta E_{n} .
$$

But any transition energy $\Delta E_{1}$ takes place in course of time $\Delta t_{1}$, the transition of $\Delta E_{2}$ is done in course of $\Delta t_{2}$, the $\Delta E_{3}$ is obtained in course of $\Delta t_{3}$, etc. So there are 


$$
\begin{aligned}
& t_{2}-t_{1}=\Delta t_{1}, \\
& t_{3}-t_{2}=\Delta t_{2}, \\
& t_{4}-t_{3}=\Delta t_{3},
\end{aligned}
$$

etc., in general

$$
t_{n+1}-t_{n}=\Delta t_{n} .
$$

Because a complementarity relation deduced from the Joule-Lenz law does exist between the elementary energy interval and transition time interval we have

$$
\Delta E_{1} \Delta t_{1}=\Delta E_{2} \Delta t_{2}=\Delta E_{3} \Delta t_{3}=\cdots=\Delta E_{n} \Delta t_{n}=h .
$$

Evidently the $t_{1}, t_{2}, t_{3}, \cdots$ entering (2) with a minus sign are the beginning times of successive intervals, and $t_{2}, t_{3}, t_{4}, \cdots$ entering with a plus sign are the end times of these intervals. In the emission process we have $\Delta E_{1}, \Delta E_{2}, \Delta E_{3}, \cdots>0$, the same property concerns by definition also the intervals $\Delta t_{1}, \Delta t_{2}, \Delta t_{3}, \cdots$

But still another kind of relations_-similar to that introduced by Kramers and Heisenberg [10]—concerns $\Delta E$ and $\Delta t$. We have

$$
\begin{aligned}
& a_{1} \Delta E_{1}=\frac{\Delta E_{1}}{\Delta t_{1}}=\frac{\left(\Delta E_{1}\right)^{2}}{h}, \\
& a_{2} \Delta E_{2}=\frac{\Delta E_{2}}{\Delta t_{2}}=\frac{\left(\Delta E_{2}\right)^{2}}{h}, \\
& a_{3} \Delta E_{3}=\frac{\Delta E_{3}}{\Delta t_{3}}=\frac{\left(\Delta E_{3}\right)^{2}}{h}, \\
& a_{4} \Delta E_{4}=\frac{\Delta E_{4}}{\Delta t_{4}}=\frac{\left(\Delta E_{4}\right)^{2}}{h}, \cdots
\end{aligned}
$$

Here $a_{1}, a_{2}, a_{3}, \cdots$ denote the transition coefficients between states 2 and 1,3 and 2, 4 and 3, etc., respectively. The coefficients lead to the emission rate represented in the last step of any formula in (4), (4a), (4b), (4c)... This step is due to application of the complementarity relation in (3).

Certainly the same relation can represent $\Delta t_{i}$ giving

$$
\frac{1}{\Delta t_{i}}=\frac{\Delta E_{i}}{h} .
$$

From the formulae in (4) it is evident that any transition coefficient $a_{i}$ satisfies the formula

$$
a_{i}=\frac{1}{\Delta t_{i}} .
$$

Simple properties of $a_{i}$ are ready to calculate. By dividing Formula (4) by (4a), (4a) by (4b), (4b) by (4c), etc., we obtain

$$
\begin{aligned}
& \frac{a_{1}}{a_{2}} \frac{\Delta E_{1}}{\Delta E_{2}}=\frac{\left(\Delta E_{1}\right)^{2}}{\left(\Delta E_{2}\right)^{2}} \rightarrow \frac{a_{1}}{a_{2}}=\frac{\Delta E_{1}}{\Delta E_{2}}, \\
& \frac{a_{2}}{a_{3}} \frac{\Delta E_{2}}{\Delta E_{3}}=\frac{\left(\Delta E_{2}\right)^{2}}{\left(\Delta E_{3}\right)^{2}} \rightarrow \frac{a_{2}}{a_{3}}=\frac{\Delta E_{2}}{\Delta E_{3}}, \\
& \frac{a_{3}}{a_{4}} \frac{\Delta E_{3}}{\Delta E_{4}}=\frac{\left(\Delta E_{3}\right)^{2}}{\left(\Delta E_{4}\right)^{2}} \rightarrow \frac{a_{3}}{a_{4}}=\frac{\Delta E_{3}}{\Delta E_{4}},
\end{aligned}
$$

etc. In effect 


$$
\begin{aligned}
& \frac{a_{1}^{2}}{a_{2}^{2}}=\frac{\left(\Delta E_{1}\right)^{2}}{\left(\Delta E_{2}\right)^{2}}=\frac{\Delta E_{1} / \Delta t_{1}}{\Delta E_{2} / \Delta t_{2}}=\frac{I_{2-1}}{I_{3-2}}, \\
& \frac{a_{2}^{2}}{a_{3}^{2}}=\frac{\left(\Delta E_{2}\right)^{2}}{\left(\Delta E_{3}\right)^{2}}=\frac{\Delta E_{2} / \Delta t_{2}}{\Delta E_{3} / \Delta t_{3}}=\frac{I_{3-2}}{I_{4-3}}, \\
& \frac{a_{3}^{2}}{a_{4}^{2}}=\frac{\left(\Delta E_{3}\right)^{2}}{\left(\Delta E_{4}\right)^{2}}=\frac{\Delta E_{3} / \Delta t_{3}}{\Delta E_{3} / \Delta t_{4}}=\frac{I_{4-3}}{I_{5-4}},
\end{aligned}
$$

etc., where

$$
I_{2-1}, I_{3-2}, I_{4-3}, I_{5-4}, \cdots
$$

are respectively the intensities, or energy rates, of transitions from level 2 to 1 , from 3 to 2 , from 4 to 3 , from 5 to 4 , etc.

We see that the ratios of the coefficient squares are equal to the ratios of intensities.

\section{Intensities in the Hydrogen Atom}

The emitted energy intensity of transition between a paricular pair of quantum states is sometimes called a component of the spectral line [11]. When expressed in energy units (ergs) per second the intensity of such line is

$$
I_{a-b}=N(a) h v A(a, b)
$$

where $N(a)$ is the number of atoms in state $a, h v$ is the energy obtained in a single electron transition, and $A(a, b)$ is the emission probability. In fact an accurate $N(a)$ is hardly possible to be estimated, $A(a, b)$ is obtainable from rather tedious quantum mechanical calculations.

The aim of the present paper is to join, as far as possible, the calculations of the emission rate of single transitions given by the present theory with the former theory of the line spectra, or obtained from experiment. Since it is difficult to make an absolute comparison between the theory, or theories, or experiment, the calculations are referred mainly to the relative intensities of the spectral lines.

In fact we shall demonstrate that the Bohr energies of electron transitions in the hydrogen atom applied in the present theory can give a rather satisfactory approximation for the ratios of the transition probabilities between the atomic states given by the quantum-mechanical theory. To this purpose the transitions from the atomic states $n_{(p)} p$ where

$$
n_{(p)}=2,3,4,5,6 \text {, }
$$

to the states $n_{(s)} s$ where

$$
n_{(s)}=1,2,3,4 \text { and } 5
$$

are considered because all results of calculations can be compared with the quantum-mechanical data listed in [11].

In examining the intensities due to the present framework we take into account the ratios

$$
\frac{I_{n_{\alpha^{\prime}} p-n_{\alpha^{\prime \prime}}}}{I_{n_{\beta^{\prime}} p-n_{\beta^{\prime \prime}}}}
$$

where

$$
n_{\alpha^{\prime}}>n_{\alpha^{\prime \prime}}
$$

and

$$
n_{\beta^{\prime}}>n_{\beta^{\prime \prime}}
$$

Both the numerator (labelled by $\alpha$ ) and denominator (labelled by $\beta$ ) entering (13) are expressed in terms 
of the Bohr energy differences

$$
\begin{aligned}
& \Delta E_{\alpha}=E_{\alpha^{\prime}}^{(p)}-E_{\alpha^{\prime \prime}}^{(s)}, \\
& \Delta E_{\beta}=E_{\beta^{\prime}}^{(p)}-E_{\beta^{\prime \prime}}^{(s)},
\end{aligned}
$$

which are positive quantities, first in view of (14) and (15), second because of the energy components equal to

$$
\begin{aligned}
& E_{\alpha^{\prime}}^{(p)}=-\frac{1}{n_{\alpha^{\prime}}^{2}} \frac{m_{e} e^{4}}{2 \hbar^{2}}, \\
& E_{\alpha^{\prime \prime}}^{(s)}=-\frac{1}{n_{\alpha^{\prime \prime}}^{2}} \frac{m_{e} e^{4}}{2 \hbar^{2}}, \\
& E_{\beta^{\prime}}^{(p)}=-\frac{1}{n_{\beta^{\prime}}^{2}} \frac{m_{e} e^{4}}{2 \hbar^{2}}, \\
& E_{\beta^{\prime \prime}}^{(s)}=-\frac{1}{n_{\beta^{\prime \prime}}^{2}} \frac{m_{e} e^{4}}{2 \hbar^{2}} .
\end{aligned}
$$

But the intensities entering (13) are represented by the formulae

$$
I_{n_{\alpha^{\prime}}(p)-n_{\alpha^{\prime \prime}}(s)}=\frac{E_{n_{\alpha^{\prime}}}^{(p)}-E_{n_{\alpha^{\prime \prime}}}^{(s)}}{t_{n_{\alpha^{\prime}}}-t_{n_{\alpha^{\prime \prime}}}^{(s)}}
$$

and

$$
I_{n_{\beta^{\prime}}(p)-n_{\beta^{\prime \prime}}(s)}=\frac{E_{n_{\beta^{\prime}}}^{(p)}-E_{n_{\beta^{\prime \prime}}}^{(s)}}{t_{n_{\beta^{\prime}}}-t_{n_{\beta^{\prime \prime}}}^{(s)}}
$$

which contain in general the time intervals different than the elementary intervals presented in (2). For example we can have

$$
t_{n_{\alpha^{\prime}}}-t_{n_{\alpha^{\prime \prime}}}=t_{5}-t_{1}
$$

or

$$
t_{n_{\beta^{\prime}}}-t_{n_{\beta^{\prime \prime}}}=t_{6}-t_{3} .
$$

However such intervals can be decomposed into elementary ones, so we obtain

$$
\begin{aligned}
t_{5}-t_{1} & =\Delta t_{4}+\Delta t_{3}+\Delta t_{2}+\Delta t_{1} \\
& =t_{5}-t_{4}+t_{4}-t_{3}+t_{3}-t_{2}+t_{2}-t_{1} \\
& =\frac{h}{\Delta E_{4}}+\frac{h}{\Delta E_{3}}+\frac{h}{\Delta E_{2}}+\frac{h}{\Delta E_{1}},
\end{aligned}
$$

or

$$
\begin{aligned}
t_{6}-t_{3} & =\Delta t_{5}+\Delta t_{4}+\Delta t_{3} \\
& =t_{6}-t_{5}+t_{5}-t_{4}+t_{4}-t_{3} \\
& =\frac{h}{\Delta E_{5}}+\frac{h}{\Delta E_{4}}+\frac{h}{\Delta E_{3}} .
\end{aligned}
$$

The last steps in the Formulaes (26) and (27) come from (3). Evidently any term entering (16) and (17) can be decomposed into $\Delta E_{i}$, for example

$$
\begin{aligned}
E_{5}^{(p)}-E_{1}^{(s)} & =E_{5}-E_{4}+E_{4}-E_{3}+E_{3}-E_{2}+E_{2}-E_{1} \\
& =\Delta E_{4}+\Delta E_{3}+\Delta E_{2}+\Delta E_{1}
\end{aligned}
$$


and

$$
\begin{aligned}
E_{6}^{(p)}-E_{3}^{(s)} & =E_{6}-E_{5}+E_{5}-E_{4}+E_{4}-E_{3} \\
& =\Delta E_{5}+\Delta E_{4}+\Delta E_{3} .
\end{aligned}
$$

On the right of (28) and (29) the indices $s$ and $p$ could be omitted because of the lack of dependence of the right-hand side of the formulae in (18)-(21) on $s(l=0)$ and $p(l=1)$.

It should be noted that for transitions between levels $n+2$ and $n$, say 3 and 1 , we arrive at a very simple intensity formula similar to those given in (4)-(4c):

$$
\frac{\Delta E_{3-1}}{\Delta t_{3-1}}=\frac{\Delta E_{2}+\Delta E_{1}}{\Delta t_{2}+\Delta t_{1}}=\frac{\Delta E_{2}+\Delta E_{1}}{\frac{h}{\Delta E_{2}}+\frac{h}{\Delta E_{1}}}=\frac{\Delta E_{2}+\Delta E_{1}}{h\left(\Delta E_{1}+\Delta E_{2}\right)} \Delta E_{1} \Delta E_{2}=\frac{\Delta E_{1} \Delta E_{2}}{h} .
$$

A time ago Einstein has remarked that the time of transitions between deep-lying quantum states should be very small [12]. The present approach does confirm this view. For example the time between $2 p$ and $1 s$ is-because of the result obtained before [6] [7] that the transition time between two neighbouring quantum levels approaches approximately the time period of the lower lying quantum state-equal to

$$
\Delta t_{1}=T_{1}=\frac{2 \pi \hbar^{3}}{m_{e} e^{4}} .
$$

This is the time period of the first quantum state in the hydrogen atom [13]. In fact the Formula (3) gives $\Delta t_{1}$ rather close to $T_{1}$ :

$$
\Delta t_{1}=\frac{h}{\Delta E_{1}}=\frac{16 \pi}{3} \frac{\hbar^{3}}{m_{e} e^{4}} \cong 4 \times 10^{-16} \mathrm{sec} .
$$

For large quantum numbers $n$ and $m$ there is evident the formula [see (6)]:

$$
\begin{aligned}
\frac{a_{m}}{a_{n}}=\frac{\Delta t_{n}}{\Delta t_{m}}=\frac{\Delta E_{m}}{\Delta E_{n}}=\frac{E_{m+1}-E_{m}}{E_{n+1}-E_{n}}=\frac{-\frac{1}{(m+1)^{2}}+\frac{1}{m^{2}}}{-\frac{1}{(n+1)^{2}}+\frac{1}{n^{2}}} \\
=\frac{\frac{(m+1)^{2}-m^{2}}{(m+1)^{2} m^{2}}}{\frac{(n+1)^{2}-n^{2}}{(n+1)^{2} n^{2}}} \cong \frac{2 m / m^{4}}{2 n / n^{4}}=\frac{n^{3}}{m^{3}},
\end{aligned}
$$

whereas the ratio of the time periods of the hydrogen atom is also:

$$
\frac{T_{n}}{T_{m}}=\frac{2 \pi n^{3} \hbar^{3}}{m_{e} e^{4}} \cdot \frac{m_{e} e^{4}}{2 \pi m^{3} \hbar^{3}}=\frac{n^{3}}{m^{3}} .
$$

Evidently the values of $\Delta t_{n} \approx T_{n}$ increase rapidly with increase of $n$.

\section{Quantum-Mechanical Counterpart of the Intensity Calculations}

The Formulaes (8)-(9) indicate a reference between the coefficients squares $a_{n}^{2}$ and $a_{n+1}^{2}$ to the intensity ratios between $I_{n+1}$ and $I_{n}$ in the sense that the ratio of the squares is equal to the ratio of intensities. This result has obtained its quantum-mechanical counterpart on the basis of the data collected in [11].

In fact we find that there exists an evident correspondence between the ratios of the quantum-mechanical transition probabilities

$$
\frac{A\left(n_{\alpha^{\prime}} p, n_{\alpha^{\prime \prime}} s\right)}{A\left(n_{\beta^{\prime}} p, n_{\beta^{\prime \prime}} s\right)}
$$


calculated for different pairs of transitions given by the $n^{\prime} p$ and $n^{\prime \prime} s$ states $\left(n^{\prime}>n^{\prime \prime}\right)$, and the intensity ratios

$$
\frac{I_{n_{\alpha^{\prime}} p-n_{\alpha^{\prime \prime}}}}{I_{n_{\beta^{\prime}} p-n_{\beta^{\prime \prime}}}}
$$

calculated with the aid of the present method. In Table 1 we present the formulae and intensity ratio (13) [or (13a)] obtained for each of the considered pair of transitions. In Table 2 the quantum-mechanical ratios of probabilities (33) calculated for each transition pair are compared with the ratios calculated in Table 1. Table 3 provides us with the abbreviated expressions for the energy intervals applied in the computations of Table 1.

In fact the ratio of two intensities obtained with the present theory referred to the corresponding ratio of the quantum-mechanical probabilities rather seldom exceeds number 2, although the ratios entering the calculations vary between the numbers being evidently smaller than unity [cases (61), (65), (86), (91)] to the numbers equal to several thousands [cases (13) and (14)].

The ratio equal to 2 is exceeded by the cases (6), (10), (31), (35), (56), (65), (86) and (91) where respectively there is obtained

$$
\begin{aligned}
\frac{\text { semiclassical ratio }}{\text { quantum-mechanical ratio }}: \frac{43}{18.4}=2.3 ; \quad \frac{90}{32}=2.8 ; \quad \frac{1.48}{0.65}=2.3 ; \quad \frac{2.08}{1.13}=2.7 ; \\
\frac{1.08}{0.49}=2.2 ; \quad \frac{0.38}{0.154}=2.5 ; \quad \frac{0.175}{0.082}=2.1 ; \quad \frac{0.081}{0.038}=2.1,
\end{aligned}
$$

but only in the case (99)

$$
\frac{\text { quantum-mechanical ratio }}{\text { semiclassical ratio }}: \frac{92.9}{41.9}=2.2
$$

exceeds 2 .

A time ago Ornstein and Burger [16] considered three intensity ratios presented in Table 1 and Table 2. These are:

$$
(4 p-2 s):(4 p-3 s),(5 p-2 s):(5 p-3 s) \text { and }(6 p-2 s):(6 p-3 s) ;
$$

see items (51), (78) and (100) in Table 1 and Table 2.

They have found respectively the following quantum-mechanical ratios for the transition probabilities:

$$
\text { 3.55, } 3.4 \text { and 3.2. }
$$

The experimental ratios of the intensities were found equal to [16]

$$
\text { 2.6, } 2.5 \text { and 2.0; }
$$

see also [11]. The data of the present theory are [see e.g. Table 1, items (51), (78) and (100)]

$$
\text { 2.86, } 2.66 \text { and 2.54, }
$$

so they are closer to the experimental data in (37) than the data given in (36).

\section{Lifetime of the Excited States}

The intensity $I_{p-q}$ of the electron transition from a state $p$ to a lower state $q$ of the hydrogen atom is coupled with the quanta of energy $\Delta E_{p-q}$ and time $\Delta t_{p-q}$ of the transition by the formula

$$
I_{p-q}=\frac{\Delta E_{p-q}}{\Delta t_{p-q}}=a_{p-q} \Delta E_{p-q} .
$$

The term $a_{p-q}$ plays the role of transition probability from $p$ to $q$; see e.g. [10].

Evidently on the basis of (39) we have

$$
\frac{1}{\Delta t_{p-q}}=a_{p-q}
$$


Table 1. Intensity ratios of electron transitions between the $p$ and $s$ states in the hydrogen atom calculated by the present method. The applied intervals of energy are listed in Table 3. A comparison of the results of the present Table with the ratios of quantum-mechanical transition probabilities is done in Table 2.

\begin{tabular}{|c|c|c|c|}
\hline No & Case & & Formula for the intensity ratio and the value of that ratio \\
\hline (1) & $\frac{2 p-1 s}{3 p-1 s}$ & $\rightarrow$ & $\frac{\left(\Delta E_{1}\right)^{2}}{\Delta E_{1} \Delta E_{2}}=\frac{\Delta E_{1}}{\Delta E_{2}}=5.4$ \\
\hline$(2)$ & $\frac{2 p-1 s}{3 p-2 s}$ & $\rightarrow$ & $\frac{\left(\Delta E_{1}\right)^{2}}{\left(\Delta E_{2}\right)^{2}}=29.2$ \\
\hline (3) & $\frac{2 p-1 s}{4 p-1 s}$ & $\rightarrow$ & $\frac{\left(\Delta E_{1}\right)^{2}}{\Delta E_{4-1}}\left(\frac{1}{\Delta E_{1}}+\frac{1}{\Delta E_{2}}+\frac{1}{\Delta E_{3}}\right)=17.1$ \\
\hline (4) & $\frac{2 p-1 s}{4 p-2 s}$ & $\rightarrow$ & $\frac{\left(\Delta E_{1}\right)^{2}}{\Delta E_{2} \Delta E_{3}}=83.3$ \\
\hline (5) & $\frac{2 p-1 s}{4 p-3 s}$ & $\rightarrow$ & $\frac{\left(\Delta E_{1}\right)^{2}}{\left(\Delta E_{3}\right)^{2}}=238$ \\
\hline (6) & $\frac{2 p-1 s}{5 p-1 s}$ & $\rightarrow$ & $\frac{\left(\Delta E_{1}\right)^{2}}{\Delta E_{5-1}}\left(\frac{1}{\Delta E_{1}}+\frac{1}{\Delta E_{2}}+\frac{1}{\Delta E_{3}}+\frac{1}{\Delta E_{4}}\right)=43$ \\
\hline (7) & $\frac{2 p-1 s}{5 p-2 s}$ & $\rightarrow$ & $\frac{\left(\Delta E_{1}\right)^{2}}{\Delta E_{5-2}}\left(\frac{1}{\Delta E_{2}}+\frac{1}{\Delta E_{3}}+\frac{1}{\Delta E_{4}}\right)=193$ \\
\hline (8) & $\frac{2 p-1 s}{5 p-3 s}$ & $\rightarrow$ & $\frac{\left(\Delta E_{1}\right)^{2}}{\Delta E_{3} \Delta E_{4}}=514$ \\
\hline (9) & $\frac{2 p-1 s}{5 p-4 s}$ & $\rightarrow$ & $\frac{\left(\Delta E_{1}\right)^{2}}{\left(\Delta E_{4}\right)^{2}}=1110$ \\
\hline (10) & $\frac{2 p-1 s}{6 p-1 s}$ & $\rightarrow$ & $\frac{\left(\Delta E_{1}\right)^{2}}{\Delta E_{6-1}}\left(\frac{1}{\Delta E_{1}}+\frac{1}{\Delta E_{2}}+\frac{1}{\Delta E_{3}}+\frac{1}{\Delta E_{4}}+\frac{1}{\Delta E_{5}}\right)=90$ \\
\hline (11) & $\frac{2 p-1 s}{6 p-2 s}$ & $\rightarrow$ & $\frac{\left(\Delta E_{1}\right)^{2}}{\Delta E_{6-2}}\left(\frac{1}{\Delta E_{2}}+\frac{1}{\Delta E_{3}}+\frac{1}{\Delta E_{4}}+\frac{1}{\Delta E_{5}}\right)=390$ \\
\hline (12) & $\frac{2 p-1 s}{6 p-3 s}$ & $\rightarrow$ & $\frac{\left(\Delta E_{1}\right)^{2}}{\Delta E_{6-3}}\left(\frac{1}{\Delta E_{3}}+\frac{1}{\Delta E_{4}}+\frac{1}{\Delta E_{5}}\right)=987$ \\
\hline (13) & $\frac{2 p-1 s}{6 p-4 s}$ & $\rightarrow$ & $\frac{\left(\Delta E_{1}\right)^{2}}{\Delta E_{4} \Delta E_{5}}=2045$ \\
\hline (14) & $\frac{2 p-1 s}{6 p-5 s}$ & $\rightarrow$ & $\frac{\left(\Delta E_{1}\right)^{2}}{\left(\Delta E_{5}\right)^{2}}=3765$ \\
\hline (15) & $\frac{3 p-1 s}{3 p-2 s}$ & $\rightarrow$ & $\frac{\Delta E_{1} \Delta E_{2}}{\left(\Delta E_{2}\right)^{2}}=\frac{\Delta E_{1}}{\Delta E_{2}}=5.4$ \\
\hline (16) & $\frac{3 p-1 s}{4 p-1 s}$ & $\rightarrow$ & $\frac{\Delta E_{1} \Delta E_{2}}{\Delta E_{4-1}}\left(\frac{1}{\Delta E_{1}}+\frac{1}{\Delta E_{2}}+\frac{1}{\Delta E_{3}}\right)=3.23$ \\
\hline (17) & $\frac{3 p-1 s}{4 p-2 s}$ & $\rightarrow$ & $\frac{\Delta E_{1} \Delta E_{2}}{\Delta E_{2} \Delta E_{3}}=\frac{\Delta E_{1}}{\Delta E_{3}}=15.4$ \\
\hline
\end{tabular}




\section{Continued}

(18)

$\frac{3 p-1 s}{4 p-3 s}$

$\frac{3 p-1 s}{5 p-1 s}$

(19)

(20)

(21)

(22)

(23)

(24)

(25)

(26)

(27)

(28)

(29)

(30)

(31)

(32)

(33)

(34)

$$
\frac{3 p-2 s}{5 p-4 s}
$$$$
\frac{3 p-1 s}{5 p-2 s}
$$$$
\frac{3 p-1 s}{5 p-3 s}
$$$$
\frac{3 p-1 s}{5 p-4 s}
$$$$
\frac{3 p-1 s}{6 p-1 s}
$$$$
\frac{3 p-1 s}{6 p-2 s}
$$$$
\frac{3 p-1 s}{6 p-3 s}
$$$$
\frac{3 p-1 s}{6 p-4 s}
$$$$
\frac{3 p-1 s}{6 p-5 s}
$$$$
\frac{3 p-2 s}{4 p-1 s}
$$$$
\frac{3 p-2 s}{4 p-2 s}
$$$$
\frac{3 p-2 s}{4 p-3 s}
$$$$
\frac{3 p-2 s}{5 p-1 s}
$$$$
\frac{3 p-2 s}{5 p-2 s}
$$$$
\frac{3 p-2 s}{5 p-3 s}
$$$$
\rightarrow
$$$$
\rightarrow
$$$$
\frac{3 p-2 s}{6 p-1 s}
$$

$$
\begin{aligned}
& \frac{\Delta E_{1} \Delta E_{2}}{\left(\Delta E_{3}\right)^{2}}=44.1 \\
& \frac{\Delta E_{1} \Delta E_{2}}{\Delta E_{5-1}}\left(\frac{1}{\Delta E_{1}}+\frac{1}{\Delta E_{2}}+\frac{1}{\Delta E_{3}}+\frac{1}{\Delta E_{4}}\right)=7.98, \\
& \frac{\Delta E_{1} \Delta E_{2}}{\Delta E_{5-2}}\left(\frac{1}{\Delta E_{2}}+\frac{1}{\Delta E_{3}}+\frac{1}{\Delta E_{4}}\right)=35.8, \\
& \frac{\Delta E_{1} \Delta E_{2}}{\Delta E_{3} \Delta E_{4}}=95.4, \\
& \frac{\Delta E_{1} \Delta E_{2}}{\left(\Delta E_{4}\right)^{2}}=205.8 \\
& \frac{\Delta E_{1} \Delta E_{2}}{\Delta E_{6-1}}\left(\frac{1}{\Delta E_{1}}+\frac{1}{\Delta E_{2}}+\frac{1}{\Delta E_{3}}+\frac{1}{\Delta E_{4}}+\frac{1}{\Delta E_{5}}\right)=16.6, \\
& \frac{\Delta E_{1} \Delta E_{2}}{\Delta E_{6-2}}\left(\frac{1}{\Delta E_{2}}+\frac{1}{\Delta E_{3}}+\frac{1}{\Delta E_{4}}+\frac{1}{\Delta E_{5}}\right)=72.2, \\
& \frac{\Delta E_{1} \Delta E_{2}}{\Delta E_{6-3}}\left(\frac{1}{\Delta E_{3}}+\frac{1}{\Delta E_{4}}+\frac{1}{\Delta E_{5}}\right)=184, \\
& \frac{\Delta E_{1} \Delta E_{2}}{\Delta E_{4} \Delta E_{5}}=379, \\
& \frac{\Delta E_{1} \Delta E_{2}}{\left(\Delta E_{5}\right)^{2}}=697, \\
& \frac{\left(\Delta E_{2}\right)^{2}}{\Delta E_{4-1}}\left(\frac{1}{\Delta E_{1}}+\frac{1}{\Delta E_{2}}+\frac{1}{\Delta E_{3}}\right)=0.60, \\
& \frac{\left(\Delta E_{2}\right)^{2}}{\Delta E_{2} \Delta E_{3}}=2.86, \\
& \frac{\left(\Delta E_{2}\right)^{2}}{\left(\Delta E_{3}\right)^{2}}=8.16, \\
& \frac{\left(\Delta E_{2}\right)^{2}}{\Delta E_{5-1}}\left(\frac{1}{\Delta E_{1}}+\frac{1}{\Delta E_{2}}+\frac{1}{\Delta E_{3}}+\frac{1}{\Delta E_{4}}\right)=1.48, \\
& \frac{\left(\Delta E_{2}\right)^{2}}{\Delta E_{5-2}}\left(\frac{1}{\Delta E_{2}}+\frac{1}{\Delta E_{3}}+\frac{1}{\Delta E_{4}}\right)=6.63, \\
& \frac{\left(\Delta E_{2}\right)^{2}}{\Delta E_{3} \Delta E_{4}}=17.6, \\
& \frac{\left(\Delta E_{2}\right)^{2}}{\left(\Delta E_{4}\right)^{2}}=38.1, \\
& \frac{\left(\Delta E_{2}\right)^{2}}{\Delta E_{6-1}}\left(\frac{1}{\Delta E_{1}}+\frac{1}{\Delta E_{2}}+\frac{1}{\Delta E_{3}}+\frac{1}{\Delta E_{4}}+\frac{1}{\Delta E_{5}}\right)=3.08,
\end{aligned}
$$




\section{Continued}

(36)

$\frac{3 p-2 s}{6 p-2 s}$

$\frac{3 p-2 s}{6 p-3 s}$

(37)

(38)

(39)

(40)

(41)

(42)

$$
\frac{3 p-2 s}{6 p-4 s}
$$$$
\frac{3 p-2 s}{6 p-5 s}
$$

$$
\frac{4 p-1 s}{4 p-2 s}
$$

$$
\frac{4 p-1 s}{4 p-3 s}
$$

$\frac{4 p-1 s}{5 p-1 s}$

(43)

(44)

(45)

(46)

(48)

(49)

$$
\frac{4 p-1 s}{5 p-2 s}
$$

$$
\frac{4 p-1 s}{5 p-3 s}
$$

$$
\frac{4 p-1 s}{5 p-4 s}
$$

$$
\frac{4 p-1 s}{6 p-1 s}
$$$$
\frac{4 p-1 s}{6 p-2 s}
$$

$$
\frac{4 p-1 s}{6 p-3 s}
$$

$$
\frac{4 p-1 s}{6 p-4 s}
$$

$\frac{\left(\Delta E_{2}\right)^{2}}{\Delta E_{6-2}}\left(\frac{1}{\Delta E_{2}}+\frac{1}{\Delta E_{3}}+\frac{1}{\Delta E_{4}}+\frac{1}{\Delta E_{5}}\right)=13.4$,

$$
\frac{\left(\Delta E_{2}\right)^{2}}{\Delta E_{6-3}}\left(\frac{1}{\Delta E_{3}}+\frac{1}{\Delta E_{4}}+\frac{1}{\Delta E_{5}}\right)=33.9,
$$$$
\frac{\left(\Delta E_{2}\right)^{2}}{\Delta E_{4} \Delta E_{5}}=70.1,
$$

$$
\frac{\left(\Delta E_{2}\right)^{2}}{\left(\Delta E_{5}\right)^{2}}=129,
$$$$
\frac{\Delta E_{4-1}}{\frac{1}{\Delta E_{1}}+\frac{1}{\Delta E_{2}}+\frac{1}{\Delta E_{3}}} \cdot \frac{1}{\Delta E_{2} \Delta E_{3}}=4.76,
$$$$
\frac{\Delta E_{4-1}}{\frac{1}{\Delta E_{1}}+\frac{1}{\Delta E_{2}}+\frac{1}{\Delta E_{3}}} \cdot \frac{1}{\left(\Delta E_{3}\right)^{2}}=13.7,
$$$$
\frac{\Delta E_{4-1}}{\frac{1}{\Delta E_{1}}+\frac{1}{\Delta E_{2}}+\frac{1}{\Delta E_{3}}} \cdot \frac{\frac{1}{\Delta E_{1}}+\frac{1}{\Delta E_{2}}+\frac{1}{\Delta E_{3}}+\frac{1}{\Delta E_{4}}}{\Delta E_{5-1}}=2.47,
$$

$$
\frac{\Delta E_{4-1}}{\frac{1}{\Delta E_{1}}+\frac{1}{\Delta E_{2}}+\frac{1}{\Delta E_{3}}} \cdot \frac{\frac{1}{\Delta E_{2}}+\frac{1}{\Delta E_{3}}+\frac{1}{\Delta E_{4}}}{\Delta E_{5-2}}=11.1,
$$

$$
\frac{\Delta E_{4-1}}{\frac{1}{\Delta E_{1}}+\frac{1}{\Delta E_{2}}+\frac{1}{\Delta E_{3}}} \cdot \frac{1}{\Delta E_{3} \Delta E_{4}}=29.5,
$$$$
\frac{\Delta E_{4-1}}{\frac{1}{\Delta E_{1}}+\frac{1}{\Delta E_{2}}+\frac{1}{\Delta E_{3}}} \cdot \frac{1}{\left(\Delta E_{4}\right)^{2}}=63.6,
$$

$$
\frac{\Delta E_{4-1}}{\Delta E_{6-1}} \cdot \frac{\frac{1}{\Delta E_{1}}+\frac{1}{\Delta E_{2}}+\frac{1}{\Delta E_{3}}+\frac{1}{\Delta E_{4}}+\frac{1}{\Delta E_{5}}}{\frac{1}{\Delta E_{1}}+\frac{1}{\Delta E_{2}}+\frac{1}{\Delta E_{3}}}=5.14,
$$

$$
\frac{\Delta E_{4-1}}{\Delta E_{6-2}} \cdot \frac{\frac{1}{\Delta E_{2}}+\frac{1}{\Delta E_{3}}+\frac{1}{\Delta E_{4}}+\frac{1}{\Delta E_{5}}}{\frac{1}{\Delta E_{1}}+\frac{1}{\Delta E_{2}}+\frac{1}{\Delta E_{3}}}=22.3,
$$

$$
\frac{\Delta E_{4-1}}{\Delta E_{6-3}} \cdot \frac{\frac{1}{\Delta E_{3}}+\frac{1}{\Delta E_{4}}+\frac{1}{\Delta E_{5}}}{\frac{1}{\Delta E_{1}}+\frac{1}{\Delta E_{2}}+\frac{1}{\Delta E_{3}}}=56.8,
$$

$\frac{\Delta E_{4-1}}{\Delta E_{4} \Delta E_{5}} \cdot \frac{1}{\frac{1}{\Delta E_{1}}+\frac{1}{\Delta E_{2}}+\frac{1}{\Delta E_{3}}}=117$, 


\section{Continued}

(50)

$\frac{4 p-1 s}{6 p-5 s}$

(51)

$$
\frac{4 p-2 s}{4 p-3 s}
$$

(52)

(53)

(54)

(55)

(56)

(57)

(58)

(59)

(60)

(61)

(62)

(63)

(64)

(65)

(66)

(67) $\frac{4 p-2 s}{5 p-1 s}$

$\frac{4 p-2 s}{5 p-2 s}$

$\frac{4 p-2 s}{5 p-3 s}$

$\frac{4 p-2 s}{5 p-4 s}$

$\frac{4 p-2 s}{6 p-1 s}$

$\frac{4 p-2 s}{6 p-2 s}$

$\frac{4 p-2 s}{6 p-3 s}$

$\frac{4 p-2 s}{6 p-4 s}$

$\frac{4 p-2 s}{6 p-5 s}$

$\frac{4 p-3 s}{5 p-1 s}$

$\frac{4 p-3 s}{5 p-2 s}$

$\frac{4 p-3 s}{5 p-3 s}$

$\frac{4 p-3 s}{5 p-4 s}$

$\frac{4 p-3 s}{6 p-1 s}$

$\frac{4 p-3 s}{6 p-2 s}$

$\frac{4 p-3 s}{6 p-3 s}$ $\rightarrow$

$\rightarrow$

$\rightarrow$

$\rightarrow$

$\rightarrow$

$\rightarrow$

$\rightarrow$

$\rightarrow$

$\rightarrow$

$\rightarrow$

$\rightarrow$

$\rightarrow$

$\rightarrow$

(2)

$\rightarrow$

$\rightarrow$

$\rightarrow$

$\rightarrow$

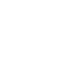

$$
\begin{gathered}
\frac{\Delta E_{4-1}}{\left(\Delta E_{5}\right)^{2}} \cdot \frac{1}{\frac{1}{\Delta E_{1}}+\frac{1}{\Delta E_{2}}+\frac{1}{\Delta E_{3}}}=216, \\
\frac{\Delta E_{2} \Delta E_{3}}{\left(\Delta E_{3}\right)^{2}}=\frac{\Delta E_{2}}{\Delta E_{3}}=2.86, \\
\frac{\Delta E_{2} \Delta E_{3}}{\Delta E_{5-1}}\left(\frac{1}{\Delta E_{1}}+\frac{1}{\Delta E_{2}}+\frac{1}{\Delta E_{3}}+\frac{1}{\Delta E_{4}}\right)=0.52, \\
\frac{\Delta E_{2} \Delta E_{3}}{\Delta E_{5-2}}\left(\frac{1}{\Delta E_{2}}+\frac{1}{\Delta E_{3}}+\frac{1}{\Delta E_{4}}\right)=2.32, \\
\frac{\Delta E_{2} \Delta E_{3}}{\Delta E_{3} \Delta E_{4}}=\frac{\Delta E_{2}}{\Delta E_{4}}=6.17,
\end{gathered}
$$

$$
\frac{\Delta E_{2} \Delta E_{3}}{\left(\Delta E_{4}\right)^{2}}=13.2
$$

$\frac{\Delta E_{2} \Delta E_{3}}{\Delta E_{6-1}}\left(\frac{1}{\Delta E_{1}}+\frac{1}{\Delta E_{2}}+\frac{1}{\Delta E_{3}}+\frac{1}{\Delta E_{4}}+\frac{1}{\Delta E_{5}}\right)=1.08$,

$$
\frac{\Delta E_{2} \Delta E_{3}}{\Delta E_{6-2}}\left(\frac{1}{\Delta E_{2}}+\frac{1}{\Delta E_{3}}+\frac{1}{\Delta E_{4}}+\frac{1}{\Delta E_{5}}\right)=4.68,
$$

$$
\frac{\Delta E_{2} \Delta E_{3}}{\Delta E_{6-3}}\left(\frac{1}{\Delta E_{3}}+\frac{1}{\Delta E_{4}}+\frac{1}{\Delta E_{5}}\right)=11.9,
$$

$$
\frac{\Delta E_{2} \Delta E_{3}}{\Delta E_{4} \Delta E_{5}}=24.6
$$

$$
\frac{\Delta E_{2} \Delta E_{5}}{\left(\Delta E_{5}\right)^{2}}=45.2
$$

$$
\frac{\left(\Delta E_{3}\right)^{2}}{\Delta E_{5-1}}\left(\frac{1}{\Delta E_{1}}+\frac{1}{\Delta E_{2}}+\frac{1}{\Delta E_{3}}+\frac{1}{\Delta E_{4}}\right)=0.18,
$$

$$
\frac{\left(\Delta E_{3}\right)^{2}}{\Delta E_{5-2}}\left(\frac{1}{\Delta E_{2}}+\frac{1}{\Delta E_{3}}+\frac{1}{\Delta E_{4}}\right)=0.81,
$$

$$
\frac{\left(\Delta E_{3}\right)^{2}}{\Delta E_{3} \Delta E_{4}}=\frac{\Delta E_{3}}{\Delta E_{4}}=2.16,
$$

$$
\frac{\left(\Delta E_{3}\right)^{2}}{\left(\Delta E_{4}\right)^{2}}=4.67
$$

$\frac{\left(\Delta E_{3}\right)^{2}}{\Delta E_{6-1}}\left(\frac{1}{\Delta E_{1}}+\frac{1}{\Delta E_{2}}+\frac{1}{\Delta E_{3}}+\frac{1}{\Delta E_{4}}+\frac{1}{\Delta E_{5}}\right)=0.38$

$$
\frac{\left(\Delta E_{3}\right)^{2}}{\Delta E_{6-2}}\left(\frac{1}{\Delta E_{2}}+\frac{1}{\Delta E_{3}}+\frac{1}{\Delta E_{4}}+\frac{1}{\Delta E_{5}}\right)=1.64,
$$

$$
\frac{\left(\Delta E_{3}\right)^{2}}{\Delta E_{6-3}}\left(\frac{1}{\Delta E_{3}}+\frac{1}{\Delta E_{4}}+\frac{1}{\Delta E_{5}}\right)=4.16,
$$




\section{Continued}

(68)

$\frac{4 p-3 s}{6 p-4 s}$

$\frac{4 p-3 s}{6 p-5 s}$

(69)

(70)

(71)

(72)

(73)

(74)

(75)

(76)

(77)

(78)

(79)

$\frac{5 p-1 s}{6 p-2 s}$

$\frac{5 p-1 s}{6 p-3 s}$

$\frac{5 p-1 s}{6 p-4 s}$

$\frac{5 p-1 s}{6 p-5 s}$

$\frac{5 p-2 s}{5 p-3 s}$

$\frac{5 p-2 s}{5 p-4 s}$

(80)

(81)

$\frac{5 p-1 s}{5 p-3 s}$

$\frac{5 p-1 s}{5 p-4 s}$

$\frac{5 p-1 s}{6 p-1 s}$

$\frac{5 p-2 s}{6 p-1 s}$ $\rightarrow$

$\frac{\Delta E_{5-1}}{\frac{1}{\Delta E_{1}}+\frac{1}{\Delta E_{2}}+\frac{1}{\Delta E_{3}}+\frac{1}{\Delta E_{4}}} \cdot \frac{1}{\Delta E_{6-1}}\left(\frac{1}{\Delta E_{1}}+\frac{1}{\Delta E_{2}}+\frac{1}{\Delta E_{3}}+\frac{1}{\Delta E_{4}}+\frac{1}{\Delta E_{5}}\right)=2.09$,

$$
\frac{\Delta E_{5-1}}{\frac{1}{\Delta E_{1}}+\frac{1}{\Delta E_{2}}+\frac{1}{\Delta E_{3}}+\frac{1}{\Delta E_{4}}} \cdot \frac{1}{\Delta E_{6-2}}\left(\frac{1}{\Delta E_{2}}+\frac{1}{\Delta E_{3}}+\frac{1}{\Delta E_{4}}+\frac{1}{\Delta E_{5}}\right)=9.05,
$$

$$
\frac{\Delta E_{5-1}}{\frac{1}{\Delta E_{1}}+\frac{1}{\Delta E_{2}}+\frac{1}{\Delta E_{3}}+\frac{1}{\Delta E_{4}}} \cdot \frac{1}{\Delta E_{6-3}}\left(\frac{1}{\Delta E_{3}}+\frac{1}{\Delta E_{4}}+\frac{1}{\Delta E_{5}}\right)=23.0,
$$$$
\frac{\Delta E_{5-1}}{\frac{1}{\Delta E_{1}}+\frac{1}{\Delta E_{2}}+\frac{1}{\Delta E_{3}}+\frac{1}{\Delta E_{4}}} \cdot \frac{1}{\Delta E_{4} \Delta E_{5}}=48.4,
$$$$
\frac{\Delta E_{5-1}}{\frac{1}{\Delta E_{1}}+\frac{1}{\Delta E_{2}}+\frac{1}{\Delta E_{3}}+\frac{1}{\Delta E_{4}}} \cdot \frac{1}{\left(\Delta E_{5}\right)^{2}}=87.4,
$$

$$
\frac{\Delta E_{5-2}}{\frac{1}{\Delta E_{2}}+\frac{1}{\Delta E_{3}}+\frac{1}{\Delta E_{4}}} \cdot \frac{1}{\Delta E_{3} \Delta E_{4}}=2.66,
$$$$
\frac{\Delta E_{5-2}}{\frac{1}{\Delta E_{2}}+\frac{1}{\Delta E_{3}}+\frac{1}{\Delta E_{4}}} \cdot \frac{1}{\left(\Delta E_{4}\right)^{2}}=5.74,
$$

$\rightarrow$

$$
\frac{\Delta E_{5-2}}{\frac{1}{\Delta E_{2}}+\frac{1}{\Delta E_{3}}+\frac{1}{\Delta E_{4}}} \cdot \frac{1}{\Delta E_{6-1}}\left(\frac{1}{\Delta E_{1}}+\frac{1}{\Delta E_{2}}+\frac{1}{\Delta E_{3}}+\frac{1}{\Delta E_{4}}+\frac{1}{\Delta E_{5}}\right)=0.46,
$$

$$
\frac{\Delta E_{5-2}}{\frac{1}{\Delta E_{2}}+\frac{1}{\Delta E_{3}}+\frac{1}{\Delta E_{4}}} \cdot \frac{1}{\Delta E_{6-2}}\left(\frac{1}{\Delta E_{2}}+\frac{1}{\Delta E_{3}}+\frac{1}{\Delta E_{4}}+\frac{1}{\Delta E_{5}}\right)=2.02,
$$




\section{Continued}

(82)

$\frac{5 p-2 s}{6 p-3 s}$

$\frac{\Delta E_{5-2}}{\frac{1}{\Delta E_{2}}+\frac{1}{\Delta E_{3}}+\frac{1}{\Delta E_{4}}} \cdot \frac{1}{\Delta E_{6-3}}\left(\frac{1}{\Delta E_{3}}+\frac{1}{\Delta E_{4}}+\frac{1}{\Delta E_{5}}\right)=5.12$,

(83)

$\frac{5 p-2 s}{6 p-4 s}$

$\frac{\Delta E_{5-2}}{\frac{1}{\Delta E_{2}}+\frac{1}{\Delta E_{3}}+\frac{1}{\Delta E_{4}}} \cdot \frac{1}{\Delta E_{4} \Delta E_{5}}=10.6$,

(84)

$\frac{5 p-2 s}{6 p-5 s}$

$\frac{\Delta E_{5-2}}{\frac{1}{\Delta E_{2}}+\frac{1}{\Delta E_{3}}+\frac{1}{\Delta E_{4}}} \cdot \frac{1}{\left(\Delta E_{5}\right)^{2}}=19.5$,

(85)

$\frac{5 p-3 s}{5 p-4 s}$

$$
\frac{\Delta E_{3} \Delta E_{4}}{\left(\Delta E_{4}\right)^{2}}=\frac{\Delta E_{3}}{\Delta E_{4}}=2.16,
$$

(86)

$\frac{5 p-3 s}{6 p-1 s}$

$\rightarrow$

$\frac{\Delta E_{3} \Delta E_{4}}{\Delta E_{6-1}}\left(\frac{1}{\Delta E_{1}}+\frac{1}{\Delta E_{2}}+\frac{1}{\Delta E_{3}}+\frac{1}{\Delta E_{4}}+\frac{1}{\Delta E_{5}}\right)=0.175$,

(87)

$\frac{5 p-3 s}{6 p-2 s}$

$\frac{\Delta E_{3} \Delta E_{4}}{\Delta E_{6-2}}\left(\frac{1}{\Delta E_{2}}+\frac{1}{\Delta E_{3}}+\frac{1}{\Delta E_{4}}+\frac{1}{\Delta E_{5}}\right)=0.76$,

(88)

$\frac{5 p-3 s}{6 p-3 s}$

$$
\frac{\Delta E_{3} \Delta E_{4}}{\Delta E_{6-3}}\left(\frac{1}{\Delta E_{3}}+\frac{1}{\Delta E_{4}}+\frac{1}{\Delta E_{5}}\right)=1.93,
$$$$
\frac{\Delta E_{3} \Delta E_{4}}{\Delta E_{4} \Delta E_{5}}=\frac{\Delta E_{3}}{\Delta E_{5}}=3.98,
$$

(90)

$$
\frac{5 p-3 s}{6 p-4 s}
$$

$$
\frac{\Delta E_{3} \Delta E_{4}}{\left(\Delta E_{5}\right)^{2}}=7.32
$$

(91)

$\frac{5 p-4 s}{6 p-1 s}$

$\frac{\left(\Delta E_{4}\right)^{2}}{\Delta E_{6-1}}\left(\frac{1}{\Delta E_{1}}+\frac{1}{\Delta E_{2}}+\frac{1}{\Delta E_{3}}+\frac{1}{\Delta E_{4}}+\frac{1}{\Delta E_{5}}\right)=0.081$,

(92)

$\frac{5 p-4 s}{6 p-2 s}$

$\frac{\left(\Delta E_{4}\right)^{2}}{\Delta E_{6-2}}\left(\frac{1}{\Delta E_{2}}+\frac{1}{\Delta E_{3}}+\frac{1}{\Delta E_{4}}+\frac{1}{\Delta E_{5}}\right)=0.35$,

(93)

$\frac{5 p-4 s}{6 p-3 s}$

$\frac{\left(\Delta E_{4}\right)^{2}}{\Delta E_{6-3}}\left(\frac{1}{\Delta E_{3}}+\frac{1}{\Delta E_{4}}+\frac{1}{\Delta E_{5}}\right)=0.89$,

(94)

$\frac{5 p-4 s}{6 p-4 s}$

$\frac{\left(\Delta E_{4}\right)^{2}}{\Delta E_{4} \Delta E_{5}}=\frac{\Delta E_{4}}{\Delta E_{5}}=1.84$,

(95)

$$
\frac{5 p-4 s}{6 p-5 s}
$$

$$
\frac{\left(\Delta E_{4}\right)^{2}}{\left(\Delta E_{5}\right)^{2}}=3.39 \text {, }
$$

(96)

$$
\frac{6 p-1 s}{6 p-2 s}
$$

$$
\frac{\Delta E_{6-1}}{\frac{1}{\Delta E_{1}}+\frac{1}{\Delta E_{2}}+\frac{1}{\Delta E_{3}}+\frac{1}{\Delta E_{4}}+\frac{1}{\Delta E_{5}}} \cdot \frac{\frac{1}{\Delta E_{2}}+\frac{1}{\Delta E_{3}}+\frac{1}{\Delta E_{4}}+\frac{1}{\Delta E_{5}}}{\Delta E_{6-2}}=4.34
$$

(97)

$\frac{6 p-1 s}{6 p-3 s} \quad \rightarrow \quad \frac{\Delta E_{6-1}}{\frac{1}{\Delta E_{1}}+\frac{1}{\Delta E_{2}}+\frac{1}{\Delta E_{3}}+\frac{1}{\Delta E_{4}}+\frac{1}{\Delta E_{5}}} \cdot \frac{\frac{1}{\Delta E_{3}}+\frac{1}{\Delta E_{4}}+\frac{1}{\Delta E_{5}}}{\Delta E_{6-3}}=11.02$




\section{Continued}

(98)

$$
\frac{6 p-1 s}{6 p-4 s}
$$

$\frac{\Delta E_{6-1}}{\frac{1}{\Delta E_{1}}+\frac{1}{\Delta E_{2}}+\frac{1}{\Delta E_{3}}+\frac{1}{\Delta E_{4}}+\frac{1}{\Delta E_{5}}} \cdot \frac{1}{\Delta E_{4} \Delta E_{5}}=22.8$,

(99)

$$
\frac{6 p-1 s}{6 p-5 s}
$$

(100)

$$
\frac{6 p-2 s}{6 p-3 s}
$$$$
\frac{6 p-2 s}{6 p-4 s}
$$

$$
\frac{6 p-3 s}{6 p-4 s}
$$

$$
\frac{6 p-2 s}{6 p-5 s}
$$$$
\frac{\Delta E_{6-1}}{\frac{1}{\Delta E_{1}}+\frac{1}{\Delta E_{2}}+\frac{1}{\Delta E_{3}}+\frac{1}{\Delta E_{4}}+\frac{1}{\Delta E_{5}}} \cdot \frac{1}{\left(\Delta E_{5}\right)^{2}}=41.9,
$$$$
\frac{\Delta E_{6-2}}{\frac{1}{\Delta E_{2}}+\frac{1}{\Delta E_{3}}+\frac{1}{\Delta E_{4}}+\frac{1}{\Delta E_{5}}} \cdot \frac{\frac{1}{\Delta E_{3}}+\frac{1}{\Delta E_{4}}+\frac{1}{\Delta E_{5}}}{\Delta E_{6-3}}=2.54,
$$

$\begin{array}{ll}\frac{6 p-3 s}{6 p-5 s} & \rightarrow \\ \frac{6 p-4 s}{6 p-5 s} & \rightarrow\end{array}$

$$
\frac{\Delta E_{6-2}}{\frac{1}{\Delta E_{2}}+\frac{1}{\Delta E_{3}}+\frac{1}{\Delta E_{4}}+\frac{1}{\Delta E_{5}}} \cdot \frac{1}{\Delta E_{4} \Delta E_{5}}=5.25,
$$$$
\frac{\Delta E_{6-2}}{\frac{1}{\Delta E_{2}}+\frac{1}{\Delta E_{3}}+\frac{1}{\Delta E_{4}}+\frac{1}{\Delta E_{5}}} \cdot \frac{1}{\left(\Delta E_{5}\right)^{2}}=9.66,
$$

\begin{tabular}{|c|c|c|c|c|}
\hline No & Case & & Quantum-mechanical ratio & Intensity ratio from Table 1 \\
\hline (1) & $\frac{2 p-1 s}{3 p-1 s}$ & $\rightarrow$ & $\frac{6.25}{1.64}=3.8$; & 5.4 \\
\hline (2) & $\frac{2 p-1 s}{3 p-2 s}$ & $\rightarrow$ & $\frac{6.25}{0.22}=28.4 ;$ & 29.2 \\
\hline (3) & $\frac{2 p-1 s}{4 p-1 s}$ & $\rightarrow$ & $\frac{6.25}{0.68}=9.4 ;$ & 17.1 \\
\hline (4) & $\frac{2 p-1 s}{4 p-2 s}$ & $\rightarrow$ & $\frac{6.25}{0.095}=65.8$ & 83.3 \\
\hline (5) & $\frac{2 p-1 s}{4 p-3 s}$ & $\rightarrow$ & $\frac{6.25}{0.030}=208$ & 238 \\
\hline (6) & $\frac{2 p-1 s}{5 p-1 s}$ & $\rightarrow$ & $\frac{6.25}{0.34}=18.4$; & 43 \\
\hline (7) & $\frac{2 p-1 s}{5 p-2 s}$ & $\rightarrow$ & $\frac{6.25}{0.049}=128$ & 193 \\
\hline
\end{tabular}$$
\frac{\Delta E_{6-3}}{\frac{1}{\Delta E_{3}}+\frac{1}{\Delta E_{4}}+\frac{1}{\Delta E_{5}}} \cdot \frac{1}{\Delta E_{4} \Delta E_{5}}=2.06
$$$$
\frac{\Delta E_{6-3}}{\frac{1}{\Delta E_{3}}+\frac{1}{\Delta E_{4}}+\frac{1}{\Delta E_{5}}} \cdot \frac{1}{\left(\Delta E_{5}\right)^{2}}=3.8,
$$

$$
\frac{\Delta E_{4} \Delta E_{5}}{\left(\Delta E_{5}\right)^{2}}=\frac{\Delta E_{4}}{\Delta E_{5}}=1.84,
$$

Table 2. Quantum-mechanical ratios of transition probabilities between the pairs of quantum levels (see [11]) compared with the intensity ratios calculated in Table 1. 


\section{Continued}

(8)

$\frac{2 p-1 s}{5 p-3 s}$

$\rightarrow$

$\frac{6.25}{0.016}=391$;

514

(9)

$$
\frac{2 p-1 s}{5 p-4 s}
$$

$$
\frac{2 p-1 s}{6 p-1 s}
$$

$$
\frac{2 p-1 s}{6 p-2 s}
$$

$$
\frac{2 p-1 s}{6 p-3 s}
$$

(16)

(26) $\rightarrow$

$\rightarrow$

$\rightarrow$

$$
\frac{3 p-1 s}{3 p-2 s}
$$

$$
\frac{2 p-1 s}{6 p-4 s}
$$$$
\frac{2 p-1 s}{6 p-5 s}
$$

$$
\frac{3 p-1 s}{4 p-1 s}
$$

$\rightarrow$

$$
\frac{3 p-1 s}{4 p-2 s}
$$

$$
\frac{3 p-1 s}{4 p-3 s}
$$

$$
\frac{3 p-1 s}{5 p-1 s}
$$

$$
\frac{3 p-1 s}{5 p-2 s}
$$

$$
\frac{3 p-1 s}{5 p-3 s}
$$

$$
\frac{3 p-1 s}{5 p-4 s}
$$

$$
\frac{3 p-1 s}{6 p-1 s}
$$$$
\frac{3 p-1 s}{6 p-2 s}
$$

$$
\frac{3 p-1 s}{6 p-3 s}
$$

$\frac{3 p-1 s}{6 p-4 s}$ $\rightarrow$

$\rightarrow$

$\rightarrow$

$\rightarrow$

$\frac{6.25}{0.0075}=833$;

$\frac{1.64}{0.030}=54.7$;

$\frac{1.64}{0.34}=4.82 ；$

$\frac{1.64}{0.049}=33.5$;

$\rightarrow \quad \frac{1.64}{0.016}=102 ;$

95.4

$\rightarrow \quad \frac{1.64}{0.0075}=218 ；$

$\frac{1.64}{0.029}=56.6$; 


\section{Continued}

(27)

$\frac{3 p-1 s}{6 p-5 s}$

$\frac{1.64}{0.0021}=781$;

697

(28)

$\frac{3 p-2 s}{4 p-1 s}$

$\frac{0.22}{0.68}=0.32$ ；

0.60

(29)

$\frac{3 p-2 s}{4 p-2 s}$

$\frac{0.22}{0.095}=2.32$;

2.86

(30)

$\frac{3 p-2 s}{4 p-3 s}$

$\frac{0.22}{0.030}=7.33 ；$

8.16

(31)

$\frac{3 p-2 s}{5 p-1 s}$

$\frac{0.22}{0.34}=0.65 ；$

1.48

(32)

$\frac{3 p-2 s}{5 p-2 s}$

$\frac{0.22}{0.049}=4.49$;

6.63

(33)

$\frac{3 p-2 s}{5 p-3 s}$

$\frac{0.22}{0.016}=13.8 ；$

17.6

(34)

$\frac{3 p-2 s}{5 p-4 s}$

$\frac{0.22}{0.0075}=29.3$;

38.1

(35)

$$
\frac{3 p-2 s}{6 p-1 s}
$$

$\frac{0.22}{0.195}=1.13$;

(36)

$$
\frac{3 p-2 s}{6 p-2 s}
$$

$\frac{0.22}{0.029}=7.6$ ；

(37)

(38)

$\frac{3 p-2 s}{6 p-3 s}$

$\frac{0.22}{0.0096}=22.9$;

$\frac{3 p-2 s}{6 p-4 s}$

$\frac{0.22}{0.0045}=48.9$;

(39)

$\frac{3 p-2 s}{6 p-5 s}$

$\frac{0.22}{0.0021}=105$;

129

(40)

$\frac{4 p-1 s}{4 p-2 s}$

$\frac{0.68}{0.095}=7.14$ ；

4.76

(41)

$\frac{4 p-1 s}{4 p-3 s}$

$\rightarrow$

$\frac{0.68}{0.030}=22.7$;

(42)

$\frac{4 p-1 s}{5 p-1 s}$

$\frac{0.68}{0.34}=2.0$;

(43)

$$
\frac{4 p-1 s}{5 p-2 s}
$$

$\frac{0.68}{0.049}=13.9$ ；

(44)

$\frac{4 p-1 s}{5 p-3 s}$

$\frac{0.68}{0.016}=42.5$ ；

(45)

$\frac{4 p-1 s}{5 p-4 s}$

$\rightarrow$

$\frac{0.68}{0.0075}=90.6$;

(46)

$\frac{4 p-1 s}{6 p-1 s}$

$\rightarrow \quad \frac{0.68}{0.195}=3.49 ;$

5.14 


\section{Continued}

(47)

$\frac{4 p-1 s}{6 p-2 s}$

$\frac{0.68}{0.029}=23.4$;

22.3

(48)

$\frac{4 p-1 s}{6 p-3 s}$

$\frac{0.68}{0.0096}=70.8$;

56.8

(49)

$\frac{4 p-1 s}{6 p-4 s}$

$\frac{0.68}{0.0045}=151$;

117

(50)

$\frac{4 p-1 s}{6 p-5 s}$

$\frac{0.68}{0.0021}=324$;

216

(51)

$\frac{4 p-2 s}{4 p-3 s}$

$\frac{0.095}{0.030}=3.17$;

2.86

(52)

$\frac{4 p-2 s}{5 p-1 s}$

$\frac{0.095}{0.34}=0.28$ ；

0.52

(53)

$\frac{4 p-2 s}{5 p-2 s}$

$\frac{0.095}{0.049}=1.94$ ；

2.32

(54)

$\frac{4 p-2 s}{5 p-3 s}$

$\frac{0.095}{0.016}=5.94$ ；

6.17

(55)

$\frac{4 p-2 s}{5 p-4 s}$

$\frac{0.095}{0.0075}=12.7$;

(56)

$\frac{4 p-2 s}{6 p-1 s}$

$\frac{0.095}{0.195}=0.49$ ；

1.08

(57)

$\frac{4 p-2 s}{6 p-2 s}$

$\frac{0.095}{0.029}=3.28$;

4.68

(58)

$\frac{4 p-2 s}{6 p-3 s}$

$\frac{0.095}{0.0096}=9.9$;

(59)

$\frac{4 p-2 s}{6 p-4 s}$

$\frac{0.095}{0.0045}=21.1$;

24.6

(60)

$\frac{4 p-2 s}{6 p-5 s}$

$\frac{0.095}{0.0021}=45.2 ＼mathrm{~ ; ~}$

45.2

(61)

$\frac{4 p-3 s}{5 p-1 s}$

$\frac{0.030}{0.34}=0.09$;

0.18

(62)

$$
\frac{4 p-3 s}{5 p-2 s}
$$

$\frac{0.030}{0.049}=0.61$;

0.81

(63)

$$
\frac{4 p-3 s}{5 p-3 s}
$$

$\frac{0.030}{0.016}=1.88$;

(64)

$$
\frac{4 p-3 s}{5 p-4 s}
$$

$\frac{0.030}{0.0075}=4.0$ ；

(65)

$\frac{4 p-3 s}{6 p-1 s}$

$\rightarrow$

$\frac{0.030}{0.195}=0.15$;

0.38

(66)

$\frac{4 p-3 s}{6 p-2 s}$

$\rightarrow \quad \frac{0.030}{0.029}=1.03$;

1.64 


\section{Continued}

(67)

$\frac{4 p-3 s}{6 p-3 s}$

$\frac{0.030}{0.0096}=3.13$;

4.16

(68)

$\frac{4 p-3 s}{6 p-4 s}$

$\frac{0.030}{0.0045}=6.67$;

8.59

(69)

$\frac{4 p-3 s}{6 p-5 s}$

$\frac{0.030}{0.0021}=14.3$

15.8

(70)

$\frac{5 p-1 s}{5 p-2 s}$

$\frac{0.34}{0.049}=6.94$;

4.49

(71)

$\frac{5 p-1 s}{5 p-3 s}$

$\frac{0.34}{0.016}=21.3$;

(72)

$\frac{5 p-1 s}{5 p-4 s}$

$\frac{0.34}{0.0075}=45.3$;

25.8

(73)

$\frac{5 p-1 s}{6 p-1 s}$

$\frac{0.34}{0.195}=1.74$;

2.09

(74)

$\frac{5 p-1 s}{6 p-2 s}$

$\frac{0.34}{0.029}=11.7$;

9.05

(75)

$$
\frac{5 p-1 s}{6 p-3 s}
$$

$\frac{0.34}{0.0096}=35.4$;

(76)

$\frac{5 p-1 s}{6 p-4 s}$

$\frac{0.34}{0.0045}=75.6$;

48.4

(77)

$\frac{5 p-1 s}{6 p-5 s}$

$\frac{0.34}{0.0021}=162$;

87.4

(78)

$\frac{5 p-2 s}{5 p-3 s}$

$\frac{0.049}{0.016}=3.06$;

2.66

(79)

$\frac{5 p-2 s}{5 p-4 s}$

$\frac{0.049}{0.0075}=6.53$;

(80)

$\frac{5 p-2 s}{6 p-1 s}$

$\frac{0.049}{0.195}=0.25$;

0.46

(81)

$\frac{5 p-2 s}{6 p-2 s}$

$\frac{0.049}{0.029}=1.69$;

2.02

(82)

$\frac{5 p-2 s}{6 p-3 s}$

$\frac{0.049}{0.0096}=5.10$;

5.12

(83)

$$
\frac{5 p-2 s}{6 p-4 s}
$$

$\frac{0.049}{0.0045}=10.9$;

10.6

(84)

$$
\frac{5 p-2 s}{6 p-5 s}
$$

$\frac{0.049}{0.0021}=23.3$

(85)

$$
\frac{5 p-3 s}{5 p-4 s}
$$

$\rightarrow$

$\frac{0.016}{0.0075}=2.13$;

(86)

$\frac{5 p-3 s}{6 p-1 s}$

$\rightarrow \quad \frac{0.016}{0.195}=0.082 ;$

0.175 


\section{Continued}

(87)

$\frac{5 p-3 s}{6 p-2 s}$

$\frac{5 p-3 s}{6 p-3 s}$

(88)

(89)

(90)

(91)

(92)

(93)

(94)

(95)

(96)

(97)

(98)

(99)

(100)

(101)

(102)

(103)

(104)

(105)

$$
\frac{5 p-3 s}{6 p-4 s}
$$$$
\frac{5 p-3 s}{6 p-5 s}
$$$$
\frac{5 p-4 s}{6 p-1 s}
$$$$
\frac{5 p-4 s}{6 p-2 s}
$$

$$
\frac{5 p-4 s}{6 p-3 s}
$$

$$
\frac{5 p-4 s}{6 p-4 s}
$$

$$
\frac{5 p-4 s}{6 p-5 s}
$$$$
\frac{6 p-1 s}{6 p-2 s}
$$$$
\frac{6 p-1 s}{6 p-3 s}
$$$$
\frac{6 p-1 s}{6 p-4 s}
$$

$\frac{6 p-1 s}{6 p-5 s}$

$\frac{6 p-2 s}{6 p-3 s}$

$\frac{6 p-2 s}{6 p-4 s}$

$$
\frac{6 p-2 s}{6 p-5 s}
$$

$\frac{6 p-3 s}{6 p-4 s}$

$\frac{6 p-3 s}{6 p-5 s}$

$\frac{6 p-4 s}{6 p-5 s}$ $\rightarrow$

$\rightarrow$

$\rightarrow$

$\rightarrow$

$\rightarrow$

$\rightarrow$

$\rightarrow$

$\rightarrow$

$\rightarrow$

$\rightarrow$

$\rightarrow$

$\rightarrow$

$\rightarrow$

$\rightarrow$

$\rightarrow$

$\rightarrow$

$\rightarrow$

$\rightarrow$

$\rightarrow$ $\frac{0.016}{0.029}=0.55$;

0.76

3.98

0.081

0.35

$\frac{0.0075}{0.029}=0.26$;

$\frac{0.0075}{0.0096}=0.78$;

0.89

$\frac{0.0075}{0.0045}=1.67$;

1.84

$\frac{0.0075}{0.0021}=3.57$;

$\frac{0.195}{0.029}=6.72$ ；

4.34

$\frac{0.195}{0.0096}=20.3$;

11.02

$\frac{0.195}{0.0045}=43.3$;

22.8

$\frac{0.195}{0.0021}=92.9$;

41.9

$\frac{0.029}{0.0096}=3.02$;

$\frac{0.029}{0.0045}=6.44$;

$\frac{0.029}{0.0021}=13.8$;

9.66

$\frac{0.0096}{0.0045}=2.10$;

2.06

$\frac{0.0096}{0.0021}=4.6$;

$\frac{0.0045}{0.0021}=2.14$; 
Table 3. Energy intervals $\Delta E$ entering the calculations of Table 1. All presented $\Delta E$ values contain the common factor of $f=m_{e} e^{4} / 2 \hbar^{2}$.

$$
\begin{gathered}
\Delta E_{1}=\left(\frac{1}{1^{2}}-\frac{1}{2^{2}}\right) f ; \quad \Delta E_{4-1}=\left(\frac{1}{1^{2}}-\frac{1}{4^{2}}\right) f ; \\
\Delta E_{2}=\left(\frac{1}{2^{2}}-\frac{1}{3^{2}}\right) f ; \quad \Delta E_{5-2}=\left(\frac{1}{2^{2}}-\frac{1}{5^{2}}\right) f ; \quad \Delta E_{6-2}=\left(\frac{1}{2^{2}}-\frac{1}{6^{2}}\right) f ; \\
\Delta E_{3}=\left(\frac{1}{3^{2}}-\frac{1}{4^{2}}\right) f ; \quad \Delta E_{6-3}=\left(\frac{1}{3^{2}}-\frac{1}{6^{2}}\right) f ; \quad \Delta E_{6-1}=\left(\frac{1}{1^{2}}-\frac{1}{6^{2}}\right) f \\
\Delta E_{4}=\left(\frac{1}{4^{2}}-\frac{1}{5^{2}}\right) f ; \quad \Delta E_{5-1}=\left(\frac{1}{1^{2}}-\frac{1}{5^{2}}\right) f ; \\
\Delta E_{5}=\left(\frac{1}{5^{2}}-\frac{1}{6^{2}}\right) f ;
\end{gathered}
$$

The lifetime of the excited state $p$ is represented by a sum of

$$
\frac{1}{a_{p-q}}=\Delta t_{p-q}
$$

performed over all possible transitions from state $p$ to states $q$ which are lower than $p$ (see [14]), i.e.

$$
E_{p}>E_{q} \text {. }
$$

In the hydrogen atom the lowest possible state $q$ is represented by $n=1$. This means that for $p=n=2$ we have only one term:

$$
\frac{1}{a_{2-1}}=\Delta t_{2-1}=\Delta t_{1}=\frac{h}{\Delta E_{1}}
$$

for $p=n=3$ we have two terms which are

$$
\frac{1}{a_{3-2}}=\Delta t_{3-2}=\Delta t_{2}=\frac{h}{\Delta E_{2}}
$$

and

$$
\frac{1}{a_{3-1}}=\Delta t_{3-1}=\Delta t_{1}+\Delta t_{2}=\frac{h}{\Delta E_{1}}+\frac{h}{\Delta E_{2}} ;
$$

for $p=n=4$ we have three transitions giving

$$
\begin{gathered}
\frac{1}{a_{4-3}}=\Delta t_{4-3}=\Delta t_{3}=\frac{h}{\Delta E_{3}}, \\
\frac{1}{a_{4-2}}=\Delta t_{4-2}=\Delta t_{3}+\Delta t_{2}=\frac{h}{\Delta E_{3}}+\frac{h}{\Delta E_{2}}, \\
\frac{1}{a_{4-1}}=\Delta t_{4-1}=\Delta t_{3}+\Delta t_{2}+\Delta t_{1}=\frac{h}{\Delta E_{3}}+\frac{h}{\Delta E_{2}}+\frac{h}{\Delta E_{1}},
\end{gathered}
$$

etc. In the last steps of (46)-(46b) we applied the partition of the transition times into their component intervals similar to those applied in Section 3.

In general the lifetime of state $p$ is

$$
T_{p}^{\text {life }}=\sum_{q} \frac{1}{a_{p-q}}
$$


where $q$ are the states lower than $p$ so they all satisfy (42). Next any reciprocal value of $a_{p-q}$ is

$$
\frac{1}{a_{p-q}}=\Delta t_{p-q}=h \sum_{i=q}^{i=p-q} \frac{1}{\Delta E_{i}} .
$$

We have found before [6] [7] that the intervals $\Delta E_{n}$ and $\Delta t_{n}$ satisfy the equation

$$
\Delta t_{n} \Delta E_{n}=h
$$

where for large $n$ the formula

$$
\Delta t_{n}=T_{n}=\frac{2 \pi \hbar^{3} n^{3}}{m e^{4}}
$$

is fulfilled with a good accuracy: the $T_{n}$ is the time period of the electron circulation about the nucleus of the hydrogen atom. Roughly the Formula (50) can be applied also for small $n$. In this way we obtain the lifetime

$$
T_{2}^{\text {life }} \cong T_{1}=\frac{2 \pi \hbar^{3}}{m e^{4}} \approx 10^{-16} \text { sec. }
$$

for the level $p=n=2$. The lifetime for the level $p=n=3$ which is a sum of (44) and (45) becomes

$$
T_{3}^{\text {life }} \cong T_{1}+2 T_{2}=\left(1^{3}+2 \times 2^{3}\right) \frac{2 \pi \hbar^{3}}{m e^{4}} ;
$$

the lifetime for the level $p=n=4$ is a sum of terms entering the Formulaes (46)-(48):

$$
T_{4}^{\text {life }} \cong T_{1}+2 T_{2}+3 T_{2}=\left(1^{3}+2 \times 2^{3}+3 \times 3^{3}\right) \frac{2 \pi \hbar^{3}}{m e^{4}} .
$$

The procedure outlined above can be extended to an arbitrary $n$. We obtain [15]

$$
T_{n}^{\text {life }} \approx \sum_{k=1}^{k=n-1} k^{4} \frac{2 \pi \hbar^{3}}{m e^{4}}=\frac{1}{30}(n-1) n(2 n-1)\left(3 n^{2}-3 n-1\right) \frac{2 \pi \hbar^{3}}{m e^{4}}
$$

so for large $n$

$$
T_{n}^{\text {life }} \approx \frac{1}{5} n^{5} \frac{2 \pi \hbar^{3}}{m e^{4}} .
$$

Evidently the present calculations do not take into account the quantum numbers other than $n$.

The quantum-mechanical calculations done for the lifetimes of the excited levels in the hydrogen atom are represented in [14]. For $n \gg 1$ this formalism gives

$$
T_{n}^{\text {life }} \sim n^{4.5} .
$$

\section{Transition Time and Emission Intensity between Energy States of the Hydrogen-Like Atom Having the Nuclear Charge $Z e ; Z>1$}

In considering the transition times of electrons in the hydrogen-like atom a situation when the electron is moving in the field of the nucleus having the charge $Z e$ where $Z>1$ seems to be of interest. In particular the change with $Z$ of the intervals $\Delta E$ and $\Delta t$ between the nearest quantum states in the system is worth to be considered. For $Z=1$ we demonstrated (see [6] [7]) that for such pairs of states the intervals of energy and time satisfy the relation

$$
\Delta E \Delta t=h .
$$

In fact both $\Delta E$ and $\Delta t$ depend on $Z$. First we have that

$$
\Delta E \sim Z^{2}
$$

because

$$
E_{n}(Z)=Z^{2} E_{n}(Z=1) \text {; }
$$


see e.g. [13]. Our aim is to calculate the dependence

$$
\Delta t=\Delta t(Z)
$$

This is an easy task if we note that the Joule-Lenz law gives

$$
\frac{\Delta E}{\Delta t}=R i^{2}
$$

where

$$
R=\frac{V}{i} \cong \frac{\Delta E}{e^{2}} T_{n}
$$

is the resistance of the current $i$ induced by the energy transition $\Delta E$ and $T_{n}$ is the time of the electron circulation involved in calculating $\Delta E$. Since

$$
i \cong \frac{e}{T_{n}}
$$

equation (61) becomes approximately

$$
\frac{\Delta E}{\Delta t} \approx \frac{\Delta E}{e^{2}} T_{n} \frac{e^{2}}{T_{n}^{2}}=\frac{\Delta E}{T_{n}}
$$

so

$$
\Delta t \approx T_{n}
$$

holds irrespectively of the size of $Z$.

It is easy to show that $T_{n}(Z)$ in a hydrogen-like system should be

$$
T_{n}(Z) \sim Z^{-2} ;
$$

see also [13]. For example let us note that according to the virial theorem

$$
2 E_{\text {kin }}^{(n)}+E_{\text {pot }}^{(n)}=0
$$

we obtain

$$
E_{n}=E_{\mathrm{kin}}^{(n)}+E_{\mathrm{pot}}^{(n)}=-E_{\mathrm{kin}}^{(n)}=\frac{1}{2} E_{\mathrm{pot}}^{(n)}
$$

where

$$
E_{\mathrm{pot}}^{(n)}=-\frac{Z e^{2}}{r_{n}(Z)} .
$$

Equation (59) together with (68) implies that

$$
r_{n}(Z)=\frac{r_{n}(Z=1)}{Z}
$$

which is in accordance with the well-known result; see e.g. [13]. On the other hand

$$
\begin{aligned}
E_{\text {kin }}^{(n)} & =\frac{m}{2} v_{n}^{2}(Z)=\frac{m}{2}\left[\frac{2 \pi r_{n}(Z)}{T_{n}(Z)}\right]^{2}=\frac{(2 \pi)^{2} r_{n}^{2}(Z=1)}{Z^{2}} \frac{1}{T_{n}^{2}(Z)} \frac{m}{2} \\
& =Z^{2} E_{\text {kin }}^{(n)}(Z=1)=Z^{2} \frac{m}{2} \frac{(2 \pi)^{2} r_{n}^{2}(Z=1)}{T_{n}^{2}(Z=1)} .
\end{aligned}
$$

This implies that

$$
Z^{4} T_{n}^{2}(Z)=T_{n}^{2}(Z=1)
$$


or

$$
T_{n}(Z)=Z^{-2} T_{n}(Z=1)
$$

which is the result given in (66).

In effect because of (65)

$$
\Delta E_{n}(Z) \Delta t_{n}(Z) \cong \Delta E_{n}(Z) \Delta T_{n}(Z)=Z^{2} \Delta E_{n}(Z=1) \frac{1}{Z^{2}} T_{n}(Z=1)=h,
$$

so the Formula (57) remains unchanged upon the change of $Z$.

But the result of (73) has an important consequence concerning the emission intensity which is

$$
I=\frac{\Delta E}{\Delta t} \cong \frac{Z^{2} \Delta E(Z=1)}{T_{n}(Z=1) \frac{1}{Z^{2}}} \cong Z^{4} \frac{\Delta E(Z=1)}{\Delta t(Z=1)},
$$

so we obtain that the intensity of transitions in the hydrogen-like system having $Z>1$ is approximately $Z^{4}$ times larger than intensity of similar transitions obtained in the hydrogen atom having $Z=1$. An experimental verification of this result seems to be a not too difficult task.

\section{Summary and Discussion}

In the paper a semiclassical approach to the transition intensities between $p$ and $s$ quantum levels of the hydrogen atom is compared with the quantum-mechanical transition probabilities for the same pairs of levels. An evident convergence between the sets of the data calculated by the both methods is obtained.

The present method is fully a non-probabilistic one. This is so because the idea of probability became unnecessary to apply as far as we do not ask when (or why) the system is going to change. In fact we look for a definite change of the occupation of quantum states in the system and the energy connected with it. In this case there is no uncertainty, or search, in the system to obtain the interval of time necessary for transition. Formally the changes of the quanta of energy and time remain on an equal footing. A difference-especially evident in the case of the hydrogen atom-is mainly connected with the computational practice: The quanta of energy are easy to calculate (with the aid of the fundamental constants of nature taken into account), but we are unable to do the same thing with the intervals of time. In effect first the intervals of energy have to be obtained, next they serve us as a background for calculating the intervals of time.

Once the system “decides” to change its definite population into another one, the time necessary to perform the transition process is defined-together with the energy change connected with transition-by the complementary relation (3), or a superposition of (3). A single (3) is adequate for an emissive transition between two neighbouring energy levels. On the other hand, if for some (unknown) reasons, the atom "decides" to choose the energy change (emission) corresponding to a larger distance between the levels than described by a single Formula (3), the transition time should necessarily fit to this requirement. In this case the individual formulae (3) serve also to calculate the components of the whole time interval necessary for transition; see formulae (26) and (27).

Computationally this makes the semiclassical approach much more simple than the quantum-mechanical one. For example we readily obtain that the ratio of the intensities $(n+1) p-n s$ transition should be larger than the intensity of $(n+2) p-(n+1) s$ transition for any $n$. This is so because the ratio of the intensity of the first kind of transitions to the intensity of the second kind transitions is given by

$$
\frac{\left(\Delta E_{n}\right)^{2}}{\left(\Delta E_{n+1}\right)^{2}}
$$

and we have (see Table 3) that

$$
\Delta E_{n}>\Delta E_{n+1}
$$

For a reason similar to (77) the intensity of $(n+2) p-n s$ should be larger than intensity $(n+3) p-(n+1) s$. This is so because the ratio of these intensities is given by 


$$
\frac{\Delta E_{n} \Delta E_{n+1}}{\Delta E_{n+1} \Delta E_{n+2}}=\frac{\Delta E_{n}}{\Delta E_{n+2}}
$$

and we have

$$
\Delta E_{n}>\Delta E_{n+2} .
$$

It should be noted that when we consider space and time as elements of a common space-and-time system, the quantum theory "selects"the time variable to a treatment based on a fully different footing than it may concern the intervals in space. Because of Equation (3) the time is divided into portions, or quanta, similar to those of their energy partners $\Delta E$.

In result a whole of the time interval between two events-which are the beginning and end of the emission-is divided into portions, or quanta, similar to those of the energy partners entering (3). In effect the time interval between two events is either elementary, i.e. defined by a single $\Delta t$ entering one of the elementary formulae in (3), or the transition process is not elementary, i.e. its time interval is composed of a sum of $\Delta t_{i}$ entering different elementary formulae in (3). A similar existence and selection of the elementary intervals defining the spatial behaviour of the electron particle seem to be yet unknown.

A simple example of an application of the Formula (30) can be given also for some cases of the ratios of the $s-p$ transition intensities which have been not yet considered in the present paper. For example we have for the ratio

$$
\frac{3 s-2 p}{4 s-2 p} \rightarrow \frac{\left(\Delta E_{2}\right)^{2}}{\Delta E_{2} \Delta E_{3}}=\frac{\Delta E_{2}}{\Delta E_{3}}=\frac{\frac{1}{2^{2}}-\frac{1}{3^{2}}}{\frac{1}{3^{2}}-\frac{1}{4^{2}}}=2.86,
$$

wheras the quantum-mechanical ratio of transition probabilities is

$$
0.63: 0.25=2.52[11][16] \text {; }
$$

for the intensity ratio

$$
\frac{4 s-3 p}{5 s-3 p} \rightarrow \frac{\left(\Delta E_{3}\right)^{2}}{\Delta E_{3} \Delta E_{4}}=\frac{\Delta E_{3}}{\Delta E_{4}}=\frac{\frac{1}{3^{2}}-\frac{1}{4^{2}}}{\frac{1}{4^{2}}-\frac{1}{5^{2}}}=2.16,
$$

whereas the quantum-mechanical ratio of transition probabilities is

$$
0.018: 0.0085=2.12[11][16]
$$

and for the intensity ratio

$$
\frac{5 s-4 p}{6 s-4 p} \rightarrow \frac{\left(\Delta E_{4}\right)^{2}}{\Delta E_{4} \Delta E_{5}}=\frac{\Delta E_{4}}{\Delta E_{5}}=\frac{\frac{1}{4^{2}}-\frac{1}{5^{2}}}{\frac{1}{5^{2}}-\frac{1}{6^{2}}}=1.84,
$$

whereas the quantum-mechanical ratio of transition probabilities is

$$
0.0065: 0.0035=1.86[11][16]
$$

\section{References}

[1] Planck, M. (1910) Acht Vorlesungen ueber Theoretische Physik. S. Hirzel, Leipzig.

[2] Einstein, A. (1917) Physikalische Zeitschrift, 18, 121-128.

[3] Van der Waerden, B.L. (1967) Sources of Quantum Mechanics. Dover, New York.

[4] Schiff, L.I. (1968) Quantum Mechanics. 3rd Edition, McGraw-Hill, New York.

[5] Bethe, H.A. and Jackiw, R.W. (1968) Intermediate Quantum Mechanics. 2nd Edition, Benjamin, New York.

[6] Olszewski, S. (2015) Journal of Modern Physics, 6, 1277-1288. http://dx.doi.org/10.4236/jmp.2015.69133 Olszewski, S. Quantum Matter. (in press) 
Olszewski, S. Reviews in Theoretical Science. (in press)

[7] Olszewski, S. (2016) Journal of Modern Physics, 7, 162-174. http://dx.doi.org/10.4236/jmp.2016.71018

[8] Kuhn, H.G. (1962) Atomic Spectra. Academic Press, New York.

[9] Bohr, N. (1918) Kgl. Danske Vid. Selsk. Scr. natur-math. Afd., 8 Raekke IV.1, Part I.

[10] Kramers, H.A. and Heisenberg, W. (1925) Zeitschrift für Physik, 31, 681-708. http://dx.doi.org/10.1007/BF02980624

[11] Condon, E.U. and Shortley, G.H. (1970) The Theory of Atomic Spectra. Cambridge University Press, Cambridge.

[12] Douglas Stone, A. (2013) Einstein and the Quantum. Princeton University Press, Princeton.

[13] Sommerfeld, A. (1931) Atombau und Spektrallinien. Vol. 1, 5th Edition, Vieweg, Braunschweig.

[14] Bethe, H. (1933) Quantenmechanik der Ein- and Zwei-Elektronenprobleme In: Geiger, H. and Scheel, K., Eds., Handbuch der Physik, Vol. 24, Part 1, Springer, Berlin, 273-560.

[15] Gradsztejn, I.S. and Ryzhik, I.M. (1963) Tables of Integrals, Sums, Series and Products. Izd. Fiziko-Matematiceskoi Literatury, Moscow. (In Russian)

[16] Ornstein, L.S. and Burger, H.C. (1930) Zeitschrift für Physik, 62, 636-639. 